\title{
Therapie der juvenilen idiopathischen Arthritis (JIA)
}

\author{
Aktualisierung der interdisziplinären AWMF-S2k-Leitlinie „Therapie der juvenilen \\ idiopathischen Arthritis“
}

\section{Therapy of juvenile idiopathic arthritis (JIA)}

\author{
Update of the interdisciplinary therapy guideline
}

Autoren

Prasad T. Oommen ${ }^{1}$, Claas Hinze ${ }^{2}$, Dirk Holzinger ${ }^{3}$, Anton Hospach ${ }^{4}$, Kirsten Minden ${ }^{5}$, Catharina Schütz

Institute

1 Heinrich-Heine-Universität Düsseldorf, Medizinische Fakultät, Klinik für Kinder-Onkologie + Hämatologie und Klinische Immunologie, Bereich Pädiatrische Rheumatologie

2 Universitätsklinikum Münster, Klinik für Pädiatrische Rheumatologie und Immunologie

3 Universitätsklinikum Essen, Klinik für Kinderheilkunde III - Bereich Pädiatrische Rheumatologie

4 Klinikum Stuttgart, Olgahospital, Zentrum für Pädiatrische Rheumatologie am Klinikum Stuttgart

5 Charité - Universitätsmedizin Berlin und Deutsches Rheuma-Forschungszentrum Berlin

6 Technische Universität Dresden, Medizinische Fakultät Carl Gustav Carus, Pädiatrie, Dresden

Schlüsselwörter

Juvenile idiopathische Arthritis, Therapie, Leitlinien

Keywords

Juvenile idiopathic arthritis, therapy, therapy guidelines

Bibliografie

DOI https://doi.org/10.1055/a-1161-5382

arthritis + rheuma 2020; 40: 260-269

(c) Georg Thieme Verlag KG Stuttgart · New York ISSN 0176-5167

Korrespondenzadresse

Dr. Prasad T. Oommen

Leiter des Bereichs Pädiatrische Rheumatologie

Heinrich-Heine Universität Düsseldorf, Medizinische Fakultät

Klinik für Kinder-Onkologie, -Hämatologie

und Klin. Immunologie

Moorenstr. 5, 40225 Düsseldorf

Prasad.Oommen@med.uni-duesseldorf.de

\section{ZUSAMMENFASSUNG}

Die Behandlung der juvenilen idiopathischen Arthritis (JIA) hat sich in den vergangenen 2 Jahrzehnten u. a. durch die Verfügbarkeit neuer Substanzen entscheidend verändert. Trotz vorhandener Zulassungen, die sich teilweise aus der Behandlung der rheumatoiden Arthritis ableiten, sind zur Einordnung der vorhandenen Evidenz Leitlinien eine wichtige Entscheidungshilfe. Die 2012 publizierte AWMF-Leitlinie zur „Therapie der juvenilen idiopathischen Arthritis“ wurde zwischen 2018 und 2019 in einem mehrstufigen Prozess aktualisiert und als S2k-Leitlinie aktualisiert. Wichtige Prinzipien und Empfehlungen zur medikamentösen und nicht-medikamentösen Therapie der JIA wurden verabschiedet. Im Fokus all dieser Maßnahmen bleibt die rasche Entzündungskontrolle, der Remissionserhalt, die Vermeidung von krankheits- und therapiebedingten Folgeschäden sowie die Gewährleistung einer störungsfreien somatischen und psychosozialen Entwicklung, Lebensqualität und Teilhabe der betroffenen Kinder und Jugendlichen.

\section{ABSTRACT}

The therapy of juvenile idiopathic arthritis (JIA) has undergone significant changes during the past two decades also due to the availability of new biologic substances. Therapy guidelines gathering the available evidence are crucial for clinical decision-making. The current interdisciplinary therapy guideline was updated between 2018 and 2019 in different steps, leading to an updated adoption of recommendations and principles of medical and non-medical therapy of JIA. The main focus and goal of all therapeutical considerations remains a rapid control of inflammation leading to and maintaining remission while avoiding disease or therapy-associated damage. This shall furthermore allow an appropriate somatic and psycho-social development along with an adequate health-related quality of life and participation of children and adolescents with JIA. 


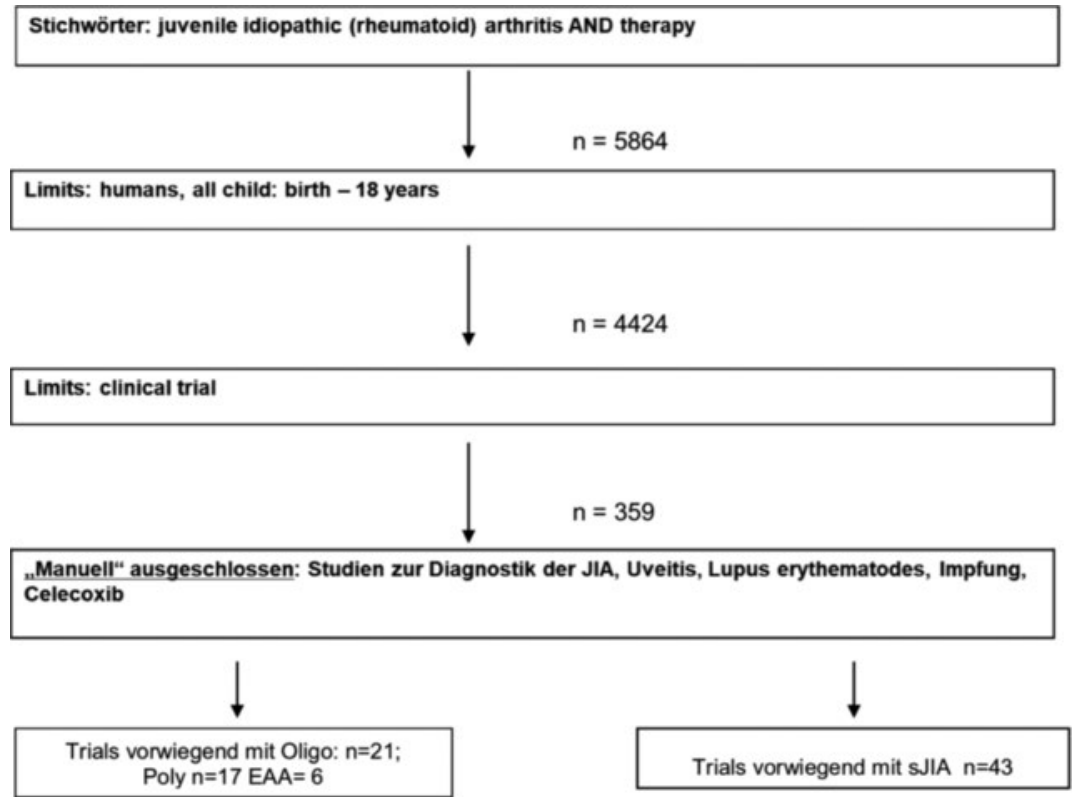

Abb. 1 Literatursuche 2007 bis 2018 verteilt auf 4 Gruppen: Oligoarthritis, Polyarthritis, Enthesitis-assoziierte Arthritis (EAA), systemische JIA (sJIA).

Die juvenile idiopathische Arthritis (JIA) ist mit einer Prävalenz von $0,1 \%$ die häufigste chronische entzündlich-rheumatische $\mathrm{Er}$ krankung im Kindes- und Jugendalter. Unter dem Begriff JIA werden nach der aktuell gültigen Klassifikation der ILAR (International League of Associations for Rheumatology) alle bei Kindern und Jugendlichen vor dem vollendeten 16. Lebensjahr auftretenden Arthritiden mit einer Dauer von mindestens 6 Wochen und unklarer Ätiologie zusammengefasst [1]. Die JIA wird klinisch diagnostiziert, diagnosebeweisende Parameter gibt es nicht. Andere, sich ähnlich präsentierende Erkrankungen, müssen ausgeschlossen werden. 6 definierte Kategorien der JIA und eine nicht definierte Gruppe (andere Arthritis) werden unterschieden. Die Kategorisierung der JIA erfolgt entsprechend der ILAR-Kriterien und basiert auf klinischen Parametern (z. B. der Zahl der in den ersten 6 Erkrankungsmonaten betroffenen Gelenke), extraartikulären Manifestationen, wenigen Laborparametern sowie der Familienanamnese.

Die Behandlung der JIA hat sich in den vergangenen $2 \mathrm{Jahr}$ zehnten entscheidend verändert. Neue krankheitsmodifizierende Medikamente (Biologika), der zunehmend frühere Einsatz von Methotrexat und die breite Anwendung von intraartikulären Glukokortikoiden gestatten eine zunehmend bessere Kontrolle der rheumatischen Entzündungsaktivität bei den betroffenen Kindern und Jugendlichen [2]. Eine inaktive Erkrankung bzw. Remission ist inzwischen das angestrebte Therapieziel und wird von der Mehrheit der Patienten (70-95\%) in den ersten Behandlungsjahren erreicht [3]. Die Änderungen im therapeutischen Vorgehen haben die Langzeitprognose der Patienten durch Abnahme von Folgeschäden und Funktionseinschränkungen im Alltag sukzessive verbessert [4]. Der therapeutische Fortschritt wurde von der Entwicklung und Validierung standardisierter Instrumente für die Bewertung des Therapieansprechens begleitet, wie z. B. des Juvenilen Arthritis Disease Activity Scores (JADAS) [5].
In den Jahren 2018-2019 wurde die bis dahin gültige AWMF-Leitlinie zur Behandlung der JIA [6] in einem mehrstufigen Prozess aktualisiert. Nach einer systematischen Literaturrecherche nach Subkategorien wurde die Leitlinie in einem interdisziplinären Prozess mit 2 Konsensuskonferenzen (Oktober 2018, März 2019) aktualisiert und nach Zustimmung aller beteiligten Fachgesellschaften und Organisationen final verabschiedet.

\section{Methoden}

Als Basis der Aktualisierung galten die Vorgängerversionen der Leitlinie aus den Jahren 2008 und 2011 [6, 7]. Somit haben die Leitlinienkoordinatoren (P. T. Oommen, C. Schütz) unterstützt durch Gruppenmitglieder (K. Minden, A. Hospach, C. Hinze, D. Holzinger) eine aktualisierte, systematische Medline-Literaturrecherche (www.ncbi.nlm.nih.gov) mit nachfolgender Suchstrategie durchgeführt: Als Stichwörter wurde „juvenile idiopathic (rheumatoid) arthritis AND therapy“; als Suchbegrenzungen (limits) „humans”, „all child 0-18 years”, „clinical trial“ verwendet. Nach manuellem Ausschluss von Studien zur Diagnostik der JIA, Uveitis, Lupus erythematodes, Impfung, Celecoxib haben die Leitlinienkoordinatoren $n=87$ Studien als Quellen hinzugezogen und hinsichtlich ihrer methodischen Qualität bewertet ( $\triangleright$ Abb. 1). Der Stichtag war der 15.01.2018.

Basierend auf diesen Studien wurden zum einen „Übergeordnete Prinzipien“ zum anderen im o. g. Konsensus-Prozess in 2 Präsenz-Konferenzen Empfehlungen zur Behandlung der juvenilen idiopathischen Arthritis formuliert (Teilnehmer siehe Kasten „Leitliniengruppe“). Die „Übergeordneten Prinzipien“ wurden in 2 Telefonkonferenzen und im E-Mail-Umlaufverfahren mit folgenden Konsensusgruppen-Mitgliedern vorbereitet: K. Minden, K. Mönkemöller, T. Kallinich, C. Schütz, P. T. Oommen. 


\section{LEITLINIENGRUPPE UND DIE DURCH DIE MITGLIEDER VERTRETENEN FACHGESELLSCHAFTEN UND ORGANISATIONEN \\ - Gesellschaft für Kinder- und Jugendrheumatologie (GKJR) Ivan Foeldvari, Gerd, Ganser, Johannes-Peter Haas, Claas Hinze, Dirk Holzinger, Anton Hospach, Hans-Iko Huppertz, Tilmann Kallinich, Ariane Klein, Kirsten Minden, Kirsten Mönkemöller, Sonja Mrusek, Ulrich Neudorf, Angelika Thon, Norbert Wagner, Catharina Schütz, Prasad T. Oommen \\ - Deutsche Rheuma-Liga, Bundesausschuss Junger Rheumatiker Karen Baltruschat \\ - Deutsche Ophthalmologische Gesellschaft (DOG) Christoph Deuter \\ - Arbeitskreis Psychosoziale Betreuung der GKJR Arnold Illhardt \\ - Deutscher Verband für Physiotherapie (ZVK e.V.) Michael Jung \\ - Deutsche Gesellschaft für Kinder- und Jugendmedizin (DGKJ) \\ Tim Niehues \\ - Deutsche Gesellschaft für Rheumatologie (DGRh) Matthias Schneider \\ - Berufsverband für Kinder- und Jugendmedizin (BVKJ) Philipp Schoof \\ - Vereinigung für Kinderorthopädie (VKO) Michael Wachowsky}

\section{Strukturierte Konsensusfindung und -prozess}

Die Konsensusfindung erfolgte im Rahmen zweier ganztägiger Konferenzen am 18.09.2018 in Mannheim (Beteiligung 100\%) und am 15.03.2019 in Düsseldorf (Beteiligung $73 \%$ ) unter der neutralen Moderation von Frau Dr. Susanne Blödt (AWMF). Zwischen den Konsensuskonferenzen wurden per digitalem Delphi-Verfahren Vorschläge für die noch offenen Empfehlungen konsentiert bzw. um Kommentare gebeten (Beteiligung 100\%).

\section{Nominale Gruppentechnik}

Die Konsensusfindung fand jeweils im Rahmen eines Nominalen Gruppenprozesses (NGP) in festlegten Schritten statt.

\section{Delphiverfahren und Konsensusstärke}

Die endgültige Abstimmung über die Empfehlungen, für welche während der 1. Konferenz noch kein Konsens erzielt werden konnte, erfolgte mittels elektronischem Delphiverfahren (E-Mail-Verteiler). Auch der überarbeitete Volltextentwurf inkl. „Übergeordneter Prinzipien“ wurde abschließend konsentiert. Adressaten waren alle Mitglieder der Leitliniengruppe. Dabei wurden folgende Angaben erfragt: Zustimmung oder keine Zustimmung oder begründete Änderungsvorschläge.

Die Ergebnisse der Befragung zu den Empfehlungen wurden zusammengefasst und bei mindestens $75 \%$ Konsens in die Leitlinie aufgenommen. Bestanden Unklarheiten bzw. Änderungswünsche, wurden diese in der 2. Konsensuskonferenz aufgegriffen und
- Tab. 1 Übergeordnete Prinzipien, die Statements zu Diagnose, Therapie, Behandlungssetting, Qualitätssicherung und Transition umfassen.

Übergeordnete Therapieprinzipien

1 Die JIA umfasst eine heterogene Gruppe von Krankheiten. Dies ist bei der Therapie zu berücksichtigen. Eine kausale Therapie ist aktuell nicht möglich.

2 Kinder und Jugendliche mit Arthritis und Verdacht auf eine JIA sollten innerhalb von 6 Wochen nach Symptombeginn einem Kinder- und Jugendrheumatologen* vorgestellt werden. Die augenärztliche Vorstellung zur Uveitis-Diagnostik soll zeitnah erfolgen.

3 Die Ziele in der Behandlung von Kindern und Jugendlichen mit JIA sind die möglichst komplette Symptom- und Entzündungskontrolle, die Verhinderung von Folgeschäden und Begleiterkrankungen, die normale Alltagsfunktion sowie die altersgerechte Entwicklung und möglichst optimale Lebensqualität der Patienten.

4 Kinder und Jugendliche sowie deren Familien sollten koordiniert, problemorientiert und interdisziplinär betreut werden. Die Therapieplanung/-koordination sollte durch einen Kinder- und Jugendrheumatologen* erfolgen.

5 Die Betreuung beinhaltet die Möglichkeit, an einer Patientenschulung teilzunehmen. Die Patienten/Familien sollten Informationen über Selbsthilfeangebote erhalten.

6 In der Behandlung sollten die aktuelle psychosoziale Situation des Kindes und seiner Familie sowie eventuelle Lebenskrisen regelmäßig erfasst und in der Therapieplanung berücksichtigt werden. Die Patienten und ihre Familien sollten einen niedrigschwelligen Zugang zu einem psychosozialen Team haben.

7 Die Festlegung der Behandlungsziele und des therapeutischen Vorgehens sollte im Rahmen einer partizipativen Entscheidungsfindung zwischen den Patienten/Eltern und dem pädiatrischen Behandlungsteam erfolgen.

8 Eine aktive JIA soll so früh wie möglich medikamentös behandelt werden.

9 Die medikamentöse Therapie richtet sich nach Aktivität, Schwere und Risikoprofil der Grunderkrankung.

10 Das Behandlungsziel besteht im frühzeitigen Erreichen und der Erhaltung einer Remission oder einer niedrigen Krankheitsaktivität („Treat-to-target“). Der Kinder- und Jugendrheumatologe steuert die Therapie anhand regelmäßiger Beurteilungen des Therapieansprechens mit krankheitsspezifischen, evaluierten Messinstrumenten.

11 Behandelnde kinder- und jugendrheumatologische Einrichtungen sollten an qualitätssichernden Maßnahmen sowie Pharmakovigilanzregistern teilnehmen.

12 Zur Sicherstellung der Behandlungskontinuität und eines bestmöglichen Outcomes sollten Jugendliche und junge Erwachsene mit JIA geplant, gut vorbereitet und mit allen notwendigen Informationen zum bisherigen Krankheitsverlauf in die internistisch- rheumatologische Weiterbetreuung übergeben werden (Transition).

* Hierbei handelt es sich um einen Kinder- und Jugendarzt mit der Zusatzbezeichnung Kinder-Rheumatologe oder einen Kinder- und Jugendarzt mit entsprechender fachlicher Erfahrung.

bis zur Abstimmungsfähigkeit angepasst. Hiernach wurde das Leitlinienmanuskript in die externe Begutachtung gegeben und hiernach abschließend in der Leitliniengruppe schriftlich abgestimmt. 
Der Gesamtentwurf der Leitlinie mit Empfehlungen wurde hiernach im starken Konsens (Zustimmung von mehr als 95\% der Teilnehmer) verabschiedet.

Alle Empfehlungen wurden im Konsens bzw. starkem Konsens angenommen. Dabei wurde die Konsensstärke wie folgt klassifiziert:

\section{Klassifikation der Konsensusstärke}

- Starker Konsens:>95\% der Stimmberechtigten

- Konsens:>75-95\% der Stimmberechtigten

- Mehrheitliche Zustimmung: > 50-75\% der Stimmberechtigten

- Dissens: $<50 \%$ der Stimmberechtigten

Die aufgeführte Konsensusstärke der Empfehlungen richtet sich auch sprachlich jeweils nach der durch die AWMF vorgegebene Klassifikation (https://www.awmf.org/leitlinien/awmf- regelwerk. html): Starke Empfehlung (soll/soll nicht), Empfehlung (sollte/ sollte nicht), Empfehlung offen (kann erwogen/verzichtet werden).

\section{Externe Begutachtung und Verabschiedung}

Das Leitlinienmanuskript wurde in die externe Begutachtung an folgende nationale und internationale Experten gegeben: Prof. Dr. K. Tenbrock, Aachen, und Prof. Dr. M. Hofer, Lausanne. Abschließend wurde die Leitlinie zwischen Oktober und November 2019 von den Vorständen der beteiligten Fachgesellschaften bzw. Organisationen formal verabschiedet.

\section{Ergebnisse}

Die Behandlung der JIA zielt auf eine rasche und effektive Entzündungskontrolle mit Remissionsinduktion/-erhalt, Vermeidung von krankheits- und/oder therapiebedingten Folgeschäden sowie die Gewährleistung einer möglichst störungsfreien somatischen und psychosozialen Entwicklung, optimalen Alltagsfunktion, Lebensqualität und Teilhabe der betroffenen Kinder und Jugendlichen.

Die Behandlungsprinzipien wurden unter 3 Aspekten betrachtet und zusammengefasst:

1. „Übergeordnete Prinzipien“: Hier wurde der Behandlungsrahmen der JIA definiert ( $\triangleright$ Tab. 1 )

2. Medikamentöse Therapie und

3. Nicht-medikamentöse Therapie.

\section{Medikamentöse Therapie}

Nach rascher Diagnosestellung sollte das Ziel der medikamentösen Therapie eine frühzeitige Kontrolle der entzündlichen Aktivität sein, um das Risiko für Folgeerkrankungen möglichst gering zu halten [8-10].

Der rechtzeitige Beginn einer Therapie beispielsweise mit Methotrexat ist mit einem besseren Ansprechen assoziiert [11]. Verschiedene Gruppen haben den Stellenwert einer frühen und intensiven („early aggressive“) Therapie einer zügig diagnostizierten JIA untersucht. Die Chance, eine klinisch inaktive Erkrankung zu erreichen, ist umso höher, je früher eine intensive Therapie beginnt [12-14].

Zur Beurteilung des Therapieansprechens stehen validierte und standardisierte Messinstrumente zur Verfügung. Die Krankheitsak- tivität wird bei der JIA anhand des JADAS (Juvenile Arthritis Disease Activity Score) bewertet [5]. Eine inaktive Erkrankung oder Remission kann anhand des JADAS und/oder der Wallace-Kriterien definiert werden [15]. Kontrollen der Krankheitsaktivität sollen bei aktiver Erkrankung regelmäßig (mindestens alle 3 Monate) mit einem evaluierten Messinstrument erfolgen, um eine zielorientierte („treat-to-target“) Therapie umsetzen zu können und die Therapie regelmäßig anzupassen [2, 5].

Zur Überwachung der Therapiesicherheit sollte ein standardisiertes Monitoring erfolgen. Demnach müssen Kinder mit JIA in regelmäßigen Abständen (üblicherweise alle 3-6 Monate) fachrheumatologisch vorgestellt werden. Insbesondere bei der Behandlung mit neu zugelassenen DMARDs (disease modifying anti-rheumatic drugs) sollten die Kinder und Jugendlichen aufgrund des noch weitgehend unbekannten Risikoprofils dieser Substanzen in der Langzeitanwendung in Pharmakovigilanzregister eingeschlossen werden.

Im Folgenden finden sich die im Zuge der Leitlinien-Aktualisierung konsentierten Empfehlungen zu den verschiedenen Stoffgruppen und Substanzen, die bei der Therapie der juvenilen idiopathischen Arthritis Einsatz finden (Kasten „Empfehlungen 1-9 (medikamentöse Therapie)“. In \ Abb. 2 findet sich die Zuordnung der jeweils empfohlenen Substanzen bezogen auf die jeweiligen JIA-Subtypen.

EMPFEHLUNGEN 1-9 (MEDIKAMENTÖSE THERAPIE)

- Empfehlung 1

NSAR sollten bei allen Subtypen der JIA zur Symptomverbesserung einer aktiven Arthritis als initiale oder begleitende Therapie eingesetzt werden.

Konsensstärke: $83 \%$

- Empfehlung 2 Eine intraartikuläre Injektion von kristalloidem Glukokortikoid (Triamcinolonhexacetonid) sollte zur Therapie der aktiven Arthritis bei JIA eingesetzt werden.

Konsensstärke: $100 \%$

- Empfehlung 3 Glukokortikoide in systemischer Applikation sollten bei hoher Krankheitsaktivität für nicht-systemische und systemische Verlaufsformen der JIA eingesetzt werden. Ein langfristiger Einsatz soll wegen unerwünschter Wirkungen und der Verfügbarkeit anderer Therapieformen nicht erfolgen.

Konsensstärke: $100 \%$

- Empfehlung 4

- Methotrexat (MTX) soll bei nicht ausreichender Wirksamkeit von NSAR, hohem oder wiederholtem Steroidbedarf oder polyartikulärer JIA eingesetzt werden. Konsensstärke: $96 \%$

- MTX kann auch bei sJIA eingesetzt werden. Konsensstärke: $96 \%$

- MTX sollte nicht zur Behandlung der isolierten axialen EAA eingesetzt werden. Konsensstärke: $80 \%$ 
Arthritis eines oder mehrerer Gelenke für $>6$ Wochen, Alter bei Beginn $<16$ Jahre, Ausschluss anderer Erkrankungen

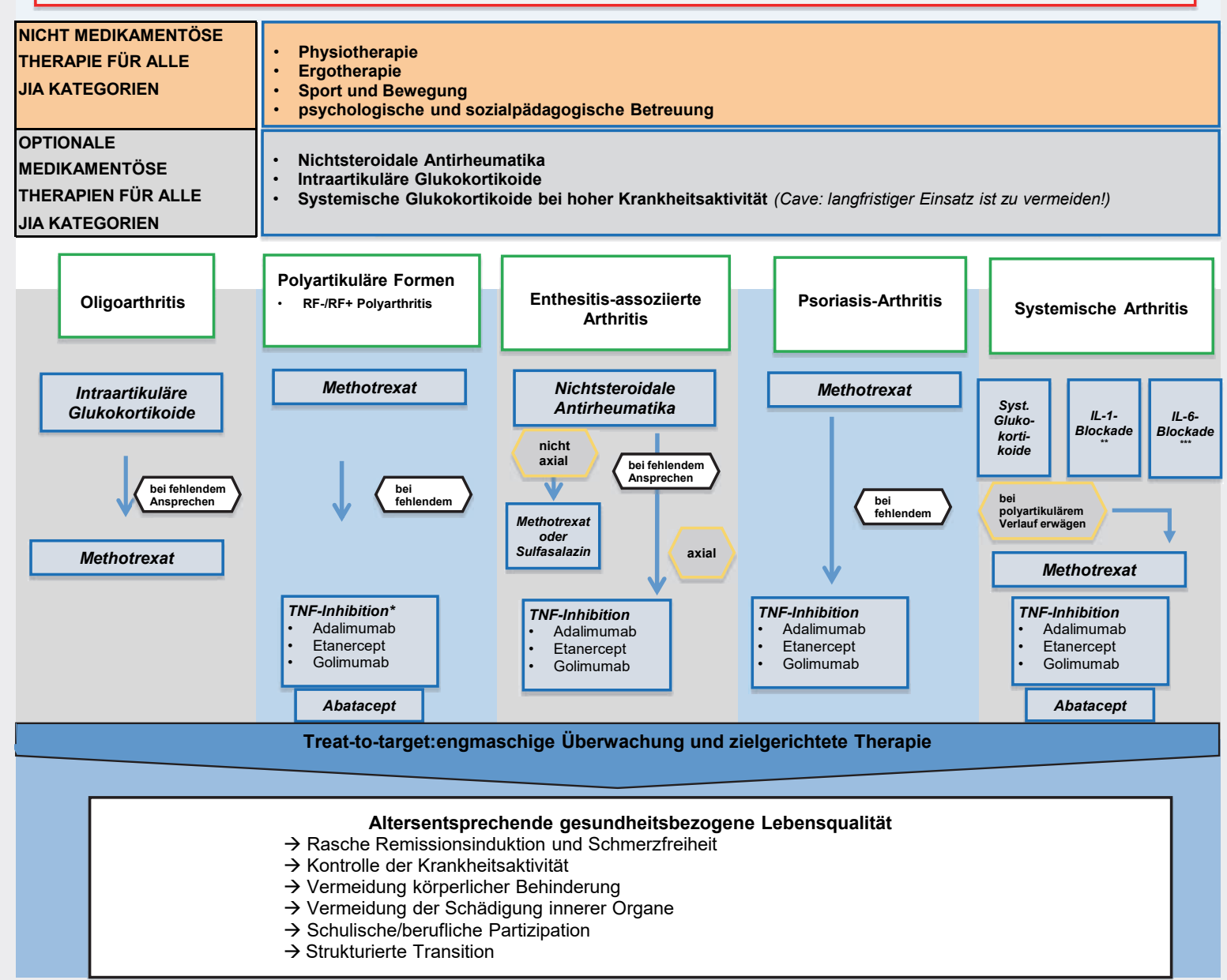

- Abb.2 Therapieempfehlung nach der AWMF-Leitlinie Therapie der juvenilen idiopathischen Arthritis: Nach Diagnosestellung auf der Basis der ILAR-Kriterien [1] kann in Abhängigkeit der verschiedenen Subtypen eine Therapie mit den dargestellten Substanzen erfolgen. Für alle Formen gelten die Empfehlungen in Hinblick auf nicht-medikamentöse Therapien; Abkürzungen: TNF= Tumornekrose-Faktor; IL-1 = Interleukin-1; IL-6 = Interleukin-6.

\section{- Empfehlung 5}

Sulfasalazin kann bei der peripheren Arthritis der

Enthesitis-assoziierten Arthritis eingesetzt werden.

Konsensstärke: $100 \%$

- Empfehlung 6

Bei unzureichendem Ansprechen oder Unverträglichkeit auf eine csDMARD-Therapie (z. B. MTX) sollte bei der nicht-systemischen JIA und kann bei der systemischen JIA TNF-alpha Inhibition eingesetzt werden. Die Wahl des TNF-Blockers sollte das Vorhandensein extraartikulärer Manifestationen berücksichtigen.

Konsensstärke: $100 \%$

- Empfehlung 7

Abatacept kann bei Patienten mit polyartikulärer JIA bei Versagen eines DMARDs eingesetzt werden.

Konsensstärke: $87 \%$

\section{- Empfehlung 8}

Tocilizumab sollte bei MTX-refraktärer polyartikulär verlaufender JIA entweder in Kombinationstherapie mit MTX oder als Monotherapie eingesetzt werden. Dies kann entweder alternativ zu einem TNF-Blocker oder nach unzureichendem Ansprechen auf einen TNF-Blocker erfolgen.

Konsensstärke: $82 \%$

- Empfehlung 9

Anakinra, Canakinumab, Glukokortikoide oder Tocilizumab sollen bei aktiver sJIA vorrangig eingesetzt werden. Eine längerfristige Therapie mit Glukokortikoiden soll vermieden werden.

Konsensstärke: $100 \%$ 


\section{Nicht-medikamentöse Therapie}

Die Pfeiler der nicht-medikamentösen Therapie der JIA umfassen eine symptomorientierte Physio- und oder Ergotherapie, körperliche Aktivität sowie eine kontinuierliche multidisziplinäre psychosoziale Versorgung der Kinder und ihrer Familien [16-18] (Kasten „Empfehlungen 10-14 (nicht-medikamentöse Therapie)“. Hierzu gehören neben psychologischer Unterstützung auch sozialpädagogische Maßnahmen wie Alltagsbewältigung, Patientenschulung, Selbstmanagement und schließlich eine strukturierte Transition in eine internistische-rheumatologische Betreuung mit Erreichen des Erwachsenenalters [19].

\section{EMPFEHLUNGEN 10-14 (NICHT-MEDIKAMENTÖSE} THERAPIE)

- Empfehlung 10 Strukturierte Behandlungen durch einen speziell geschulten bzw. spezialisierten Physiotherapeuten/Ergotherapeuten in Kombination mit der medikamentösen Therapie sollen bei Bedarf erfolgen, um die Gelenkbeweglichkeit zu erhalten bzw. zu verbessern. Anleitungen zu individuellen, täglich durchzuführenden Übungsprogrammen im häuslichen Umfeld sollen in das Therapiekonzept integriert werden. Speziell angefertigte Hilfsmittel bei Achsenabweichungen oder Wachstumsverzögerung können zur Vermeidung von Fehlbelastungen, Stabilisierung der Gelenke (z. B. Hand-, Finger-, Fußorthesen) und zur Normalisierung der Bewegungsmuster empfohlen und ärztlich verordnet werden.

Konsensstärke: $86 \%$

- Empfehlung 11

Der Einsatz physikalischer Therapie (u. a. Thermotherapie, Elektrotherapie, Massage und Lymphdrainage) kann erwogen werden.

Konsensstärke: $86 \%$

- Empfehlung 12

Kinder und Jugendliche mit JIA sollen zu einem aktiven Lebensstil und zu sportlichen Aktivitäten ermutigt werden.

Konsensstärke: $93 \%$

- Empfehlung 13 Nach ausbleibendem Erfolg der konservativen Therapiemaßnahmen oder Komplikationen (z. B. bei Achsfehlern, bei Beinlängendifferenzen oder Gelenkdestruktion) sollten Kinderorthopäden hinzugezogen werden.

Konsensstärke: 94\%

- Empfehlung 14

Eine psychologische Betreuung in der pädiatrisch-rheumatologischen Regelversorgung soll bei Bedarf zu einem frühen Zeitpunkt erfolgen, um psychische Prozesse und spezifische Verhaltensauffälligkeiten zu erfassen, aufzufangen und zu behandeln.

Konsensstärke: $83 \%$

\section{Diskussion}

In einem aufwendigen, mehrstufigen Prozess wurde die AWMF-Leitlinie zur Therapie der juvenilen idiopathischen Arthritis aktualisiert. Auch im internationalen Vergleich ist der breite methodische Ansatz der Leitlinie in Hinblick auf die vorangehende systematische Literaturrecherche sowie die interdisziplinäre Konsensusgruppen-Zusammensetzung hervorzuheben.

In den vergangenen 2 Jahrzehnten haben sich die medikamentösen Optionen bei der Behandlung der juvenilen idiopathischen Arthritis deutlich erweitert. Das Erreichen von Entzündungsfreiheit und Remission sind ein realistisches und erreichbares Therapieziel geworden, womit sich Lebensqualität und Teilhabe von Kindern und Jugendlichen mit JIA deutlich verbessert haben [2, 20-23].

Im Gegensatz zu international zunehmend verbreiteten und auch in Deutschland vorgeschlagenen Consensus Treatment Plans (CTP), bei denen verschiedene Therapieansätze für die Behandlung definierter rheumatischer Erkrankungen vorgeschlagen und geprüft werden, verfolgt die Leitlinienarbeit ein anderes Ziel [2427]. Es wird die vorhandene Evidenz für z. T. etablierte oder auch zugelassene Behandlungsmodalitäten in den Fokus genommen, während die o.g. CTP keine Placebo-kontrollierten Effektivitätsprüfungen einzelner Substanzen vornehmen, sondern verschiedene Behandlungsansätze prüfen.

Zwischen dem Ende der Literaturrecherche der 2. Auflage der JIA-Leitlinie (15.01.2010) wurden mit Tocilizumab, Anakinra, Canakinumab und Golimumab allein 4 Substanzen zur Behandlung spezieller Subtypen der JIA zugelassen. Vor diesem Hintergrund war die Aktualisierung und Evidenz-basierte Recherche dringend erforderlich.

Im Ergebnis wurden zunächst 12 sogenannte „Übergeordnete Prinzipien“, die Aussagen zu Diagnose, Therapie, Behandlungssetting, Qualitätssicherung und Transition den eigentlichen Therapieempfehlungen voranstellen, entwickelt. Hier konnte der Therapierahmen, der für eine zeitgemäße Behandlung rheumakranker Kinder und Jugendlicher und ihrer Familien angemessen erscheint, skizziert werden. Wesentlich und unstrittig ist beispielsweise, dass die Behandlung der Patienten mit JIA in die Hände erfahrener Kinder- und Jugendrheumatologen in einem kompetenten, multidisziplinären Behandlungsteam gehört. Ein solches Team ist erforderlich für eine passgenaue Therapiesteuerung der medikamentösen, nicht-medikamentösen und psychosozialen Betreuung dieser Patienten.

Die folgenden Empfehlungen zur medikamentösen Therapie der JIA berücksichtigen die aktuell eingesetzten Substanzen, von NSAR über Glukortikoide, cs- (konventionell-synthetischen) DMARds bis hin zu b- (biologischen) DMARDs. Die niedrige Evidenz für häufig eingesetzte Substanzen wie NSAR lässt sich an der hierzu kontroversen Diskussion in der Konsensusgruppe und der vergleichsweise niedrigen Konsenstärke von 83 \% ablesen. Andererseits fand sich bei guter Evidenz ein hoher Konsens für die bei der systemischen juvenilen idiopathischen Arthritis eingesetzten IL-1- und IL-6-blockierenden Substanzen und Glukokortikoide mit einer $100 \%$ Konsensstärke.

Im Vergleich zur Vorläufer-Version der JIA-Leitlinie ist neben einer Aktualisierung der Evidenz für die Substanzen und den Übergeordneten Prinzipien ein deutlicher Schwerpunkt auf die Konsen- 
tierung der nicht-medikamentösen Therapien gelegt worden. Auch hier musste in Ermangelung hoher Evidenz vor allem im Konsensusprozess Einigkeit erzielt werden. Die aktive Teilnahme von Experten aus den Bereichen Psychologie, Physiotherapie, Sport und Selbsthilfe waren hier von besonderem Wert und unterscheiden diese Leitlinie auch von anderen, eher ärztlich dominierten, Formaten. So konnte ein klares Statement zum hohen Stellenwert eines aktiven Lebensstils und sportlicher Aktivität bei rheumakranken Kindern und Jugendlicher verabschiedet werden.

Die Nachteile einer Evidenz-basierten Leitlinie liegen im Prinzip der oben beschriebenen Rückschau: Die Literaturrecherche endet für die vorliegende Arbeit im Jahr 2018, der Abschluss des aufwendigen Konsensusprozesses war im Jahr 2019. Arbeiten, die seit dem Ende der Literaturrecherche erschienen sind, konnten aus methodischen Gründen nicht in die Bewertung aufgenommen werden. Somit ist eine Leitlinie, so auch die hier vorliegende, zum Zeitpunkt der Publikation und Implementierung bereits nicht mehr auf dem aktuellen Stand. In diesem vielleicht konservativ und etwas starr wirkenden Leitlinien-Prozess liegt andererseits auch ihre Stärke. Die Aussagekraft und der Empfehlungsgrad reflektieren durch seine Evidenz-Bezogenheit das große Maß an Verantwortung, das bei der Behandlung von Kindern und Jugendlichen mit innovativen, allerdings auch immunologisch-invasiven Substanzen zum Tragen kommt.

Viele praktische Fragen des therapeutischen Alltags kann die Leitlinie nicht beantworten, da hierfür ausreichende Evidenz nicht zur Verfügung steht. Zentral ist hier sicher die Frage nach dem Zeitpunkt (Mindestdauer der „Remission unter Medikation“) und der Art der Therapiedeeskalation und -beendigung (Ausschleichen vs. abruptem Absetzen). Erste Hinweise liegen vor und können Entscheidungshilfen sein.

Zusammenfassend bildet die hier vorliegende Aktualisierung der JIA-Leitlinie mit 12 übergeordneten Therapieprinzipien, 9 Empfehlungen zur medikamentösen und 5 Empfehlungen zur nicht-medikamentösen Therapie der JIA die aktuelle Evidenz-basierte Datenlage ab. Bei fehlender oder unzureichender Evidenz konnte auf der Basis der S2k-Leitlinien-Prinzipien durch 2 moderierte Konsensuskonferenzen ein interdisziplinärer Konsens erzielt werden. Die kontinuierliche und kritische Analyse der Evidenz und die Aktualisierung dieser Leitlinie ist auch zukünftig wichtig, um im Ergebnis einen Beitrag zur rationalen und verantwortungsbewussten Behandlung rheumakranker Kinder und Jugendlicher zu leisten.
Interessenkonflikt
$\mathrm{CH}$ : Honorare von Novartis

DH: Honorare von Novartis, Sobi

AH: Advisory Boards Novartis, Sobi

KM: Honorare von AbbVie, gsk, medac, Roche und Sanofi

CS: Novartis (Forschungsförderung)

PTO: Novartis (Forschungsförderung)

Finanzierung und redaktionelle Unabhängigkeit: Die Aktualisierung der Leitlinie entstand ohne Einflussnahme oder finanzielle Unterstützung von Sponsoren. Finanzielle Unterstützung erfolgte ausschließlich durch die Gesellschaft für Kinder- und Jugendrheumatologie (GKJR) sowie durch eine spezielle Leitlinien-Förderung der Deutschen Gesellschaft für Kinder- und Jugendmedizin (DGKJ). Diese Mittel wurden eingesetzt für die im Rahmen der Planungstreffen und Konsensusverfahren angefallenen Kosten (Reisekosten und Verpflegung) sowie Kosten für Büromaterial. Alle Mitglieder der Leitliniengruppe legten eine schriftliche Erklärung zu eventuell bestehenden Interessenkonflikten insbesondere gegenüber der Industrie sowie indirekte Interessen auf dem AWMF-Formblatt 2018 vor.

Danksagung

Wir danken Dr. Susanne Blödt für die unermüdliche inhaltliche und redaktionelle Unterstützung des gesamten Leitlinien-Prozesses. Für die Unterstützung im Leitlinien-Sekretariat danken wir sehr herzlich Frau Sabine Schmitz.

\section{Literatur}

[1] Petty RE, Southwood TR, Manners P et al. International League of Associations for Rheumatology. J Rheumatol 2004; 31 (2): 390-392

[2] Ravelli A, Consolaro A, Horneff G et al. Treating juvenile idiopathic arthritis to target: recommendations of an international task force. Ann Rheum Dis 2018; 77: 819-828

[3] Hissink Muller P, Brinkman DMC, Schonenberg-Meinema D et al. Treat to target (drug-free) inactive disease in DMARD-naive juvenile idiopathic arthritis: 24-month clinical outcomes of a three-armed randomised trial. Ann Rheum Dis 2019; 78 (1): 51-59

[4] Minden K, Horneff G, Niewerth M et al. Time of Disease-Modifying Antirheumatic Drug Start in Juvenile Idiopathic Arthritis and the Likelihood of a Drug-Free Remission in Young Adulthood. Arthritis Care Res (Hoboken) 2019; 71 (4): 471-481

[5] Consolaro A, Ruperto N, Bazso A et al. Development and validation of a composite disease activity score for juvenile idiopathic arthritis. Arthritis Rheum 2009; 61: 658-666

[6] Dueckers G, Guellac N, Arbogast M et al. Evidence and consensus based GKJR guidelines for the treatment of juvenile idiopathic arthritis. Clin Immunol 2012; 142: 176-193

[7] Guellac N, Niehues T. Interdisziplinäre S2-Therapieleitlinie der Juvenilen idiopathischen Arthritis. Klin Padiatr 2008; 220 (6): 392-402

[8] Magnani A, Pistorio A, Magni-Manzoni S et al. Achievement of a state of inactive disease at least once in the first 5 years predicts better outcome of patients with polyarticular juvenile idiopathic arthritis. J Rheumatol 2009; 36: 628-634

[9] Simard JF, Neovius M, Hagelberg S et al. Juvenile idiopathic arthritis and risk of cancer: a nationwide cohort study. Arthritis Rheum 2010; 62: $3776-3782$ 
[10] Shoop-Worrall SJW, Kearsley-Fleet L, Thomson W et al. How common is remission in juvenile idiopathic arthritis: A systematic review. Semin Arthritis Rheum 2017; 47 (3): 331-337

[11] Cellucci T, Guzman J, Petty RE et al. Management of Juvenile Idiopathic Arthritis 2015: a position statement from the pediatric Committee of the Canadian Rheumatology Association. J Rheumatol 2016; 43 (10): 1773-1776

[12] Wallace CA, Giannini EH, Spalding SJ et al. Trial of Early Agressive Therapy in polyarticular juvenile idiopathic arthritis. Arthritis Rheum 2012; 64 (6): 2012-2021

[13] Wallace CA, Ringold S, Bohnsack J et al. Extension study of participants from the trial of early aggressive therapy in juvenile idiopathic arthritis. J Rheumatol 2014; 41 (12): 2459-2465

[14] Hissink Muller PCE, Brinkman DMC, Schonenberg D et al. A comparison of three treatment stratgies in recent onset non-systemic juvenile idiopathic arthritis: initial 3-months results of the best for kids-study. Pediatric Rheumatology 2017; 15: 11

[15] Wallace CA, Ruperto N, Giannini E et al. Preliminary criteria for clinical remission for select categories of juvenile idiopathic arthritis. J Rheumatol 2004; 31 (11): 2290-2294

[16] Singh-Grewal D, Wright V, Bar-Or O et al. Pilot study of fitness training and exercise testing in polyarticular childhood arthritis. Arthritis Rheum 2006; 55: 364-372

[17] Lelieveld OT, Armbrust W, van Leeuwen MA et al. Physical Activity in Adolescents With Juvenile Idiopathic Arthritis. Arthritis Rheum 2008; 59 (10): 1379-1384

[18] Erhart M, Weimann A, Bullinger M et al. Psychische Komorbidität bei chronisch somatischen Erkrankungen im Kindes- und Jugendalter. Bundesgesundheitsblatt-Gesundheitsforschung-Gesundheitsschutz 2011; 54 (1): 66-74
[19] Foster HE, Minden K, Clemente D et al. EULAR/PReS standards and recommendations for the transitional care of young people with juvenile-onset rheumatic diseases. Ann Rheum Dis 2017; 76 (4): 639-646

[20] Guzman J, Oen K, Tucker LG et al. The outcomes of juvenile idiopathic arthritis in children managed with contemporary treatments: results from the ReACCh-Out cohort. Ann Rheum Dis 2015; 74 (10): $1854-1860$

[21] Glerup M, Herlin T, Twilt M. Clinical Outcome and Long-term Remission in JIA. Curr Rheumatol Rep 2017; 19 (12): 75

[22] Giancane G, Muratore V, Marzetti V et al. Disease activity and damage in juvenile idiopathic arthritis: methotrexate era versus biologic era. Arthritis Res Ther 2019; 21 (1): 168

[23] Minden K, Horneff G, Niewerth M et al. Time of Disease-Modifying Antirheumatic Drug Start in Juvenile Idiopathic Arthritis and the Likelihood of a Drug-Free Remission in Young Adulthood. Arthritis Care Res (Hoboken) 2019; 71 (4): 471-481

[24] Ringold S, Weiss PF, Colbert RA et al. Childhood arthritis and rheumatology research alliance consensus treatment plans for new-onset polyarticular juvenile idiopathic arthritis. Arthritis Care Res (Hoboken) 2014; 66 (7): 1063-1072

[25] Hinze CH, Holzinger D, Lainka E et al. Practice and consensus-based strategies in diagnosing and managing systemic juvenile idiopathic arthritis in Germany. Pediatr Rheumatol Online J 2018; 16: 7

[26] Horneff G, Klein A, Ganser G et al. Protocols on classification, monitoring and therapy in children's rheumatology (PRO-KIND). Results of the working group polyarticular juvenile idiopathic arthritis. Pediatr Rheumatol Online J 2017; 15: 78

[27] Oommen PT, Niehues T. Sind die Leitlinien das non plus ultra? Anwendung von Leitlinien bei der JIA im klinischen Alltag. Akt Rheumatol 2015; 40: 280-283 